\title{
Normal Mode Relaxation in Linear and Branched Polyisoprene
}

\author{
C. M. Roland* and C. A. Bero
}

Chemistry Division, Code 6120, Naval Research Laboratory, Washington, D.C. 20375-5342

Received J anuary 23, 1996; Revised Manuscript Received May 21, $1996^{\otimes}$

\begin{abstract}
Dielectric spectroscopy was carried out on linear and three-arm star polyisoprenes (PI). The shape of the normal mode peak differed significantly from the corresponding mechanical terminal relaxation function, although the temperature dependencies measured for the two spectroscopies were similar. This represents a departure from the usual correlation between time and temperature dependencies. In accord with mechanical relaxation data on these same polymers, the normal mode peak is found to be broader and more sensitive to temperature for star-branched PI than for the linear polymer.
\end{abstract}

\section{Introduction}

Dielectric spectroscopy is a useful compliment to the more customary mechanical methods of probing the viscoelastic properties of polymers. While many dielectric studies have been carried out on local segmental motion and secondary relaxations in polymers, ${ }^{1,2}$ investigation of the terminal, or "normal mode", relaxation is less common. The main reason for this is that motions associated with the "chain modes" only affect the dielectric response for polymers with a dipole moment parallel to the chain contour ("type-A" polymers). In the usual case, wherein the dipole moment of a type-A polymer is sufficiently weak such that dipole-dipole correlations are negligible, the normal mode directly reflects the motion of the end-to-end chain vector. ${ }^{3}$

The dielectric behavior of the type-A polymer 1,4polyisoprene (PI) has been extensively studied. Early work employed natural rubber 4,5 and was thus limited to investigations of segmental relaxation, since the normal mode for material of such high molecular weight is obscured by conductivity. More recently, Adachi and coworkers published a series of papers describing dielectric measurements on both the segmental and terminal modes of PI.6,7 They established that the molecular weight dependence of the dielectric relaxation times was essentially the same as that of the corresponding mechanical relaxation times. They also observed some increase in the breadth of the normal mode peak with increasing molecular weight.

A number of investigations have been carried out on the dielectric response of PI dispersed in polybutadiene matrices. ${ }^{8-12}$ Such studies can provide unique information, because the polybutadiene, lacking any dipole moment parallel to the chain direction, does not interfere with the PI normal mode. When the matrix molecular weight is very high, the probe $\mathrm{PI}$ chains relax much faster than the surrounding medium; that is, constraint release and tube renewal mechanisms are suppressed. This allows for stringent tests of theoretical models for the low frequency dynamics of polymers. ${ }^{9,10}$

Boese and Kremer ${ }^{13,14}$ carried out dielectric measurements on linear and branched polyisoprenes, quantifying the dependence of the breadth of the normal mode peak on molecular weight. They also reported that, while the normal mode for star-branched polyisoprene is shifted to lower frequencies relative to the linear

\footnotetext{
${ }^{\otimes}$ Abstract published in Advance ACS Abstracts, October 15, 1996.
}

polymer, the respective temperature dependencies were similar. 14

Although reorientation of the end-to-end vector of a type-A polymers results in a normal mode relaxation corresponding to the terminal relaxation observed mechanically, the dielectric technique is not as generally useful. In the frequency regime of most dielectric spectrometers $(>10 \mathrm{~Hz})$, the normal mode response is often masked to some degree by sample conductivity. Interference from the latter is worse for higher molecular weight polymers, since their spectra must be obtained at higher temperatures in order to bring the normal mode into the experimentally accessible range of frequencies. It is for this reason that most of the published dielectric data for the normal mode is limited to low molecular weight polymers. ${ }^{6-14}$

This problem can be avoided by obtaining dielectric measurements at lower frequencies, using a time domain instrument. This has the additional benefit of providing data at the same frequencies and temperatures as mechanical spectrometers. In the present study, the normal mode relaxation of linear and starbranched polyisoprene was characterized and contrasted with previously reported mechanical spectroscopy results on the same polymers. The low-frequency range of the dielectric measurements enabled the use of samples higher in molecular weight than previous work. ${ }^{6-14}$ This is helpful in isolating the terminal response from the shorter time dynamics; moreover, for our purposes, it is necessary to use star $\mathrm{PI}$ for which the branches are highly entangled. When this is the case, the terminal viscosity, which for branched polymers depends primarily on the molecular weight of the arms rather than the total molecular weight, will exceed that of the corresponding linear polymer. In this entangled regime, the specific consequences of branching on the rheology become prominent.

\section{Experimental Section}

The polyisoprenes were 93\% 1,4-microstructure (Polymer Source, Inc., Quebec, Canada), prepared by anionic polymerization using sec-butyllithium as the initiator. The three-arm star, formed by coupling the linear polymer using 2,4,6-tris(allyloxy)-1,3,5-triazine, was repeatedly fractionated in a benzene/methanol solution prior to use. These polymers are identical to those used in earlier dynamic mechanical testing. ${ }^{15}$ Their molecular weights and the distributions are listed in Table 1.

Dielectric experiments employed a time domain spectrometer (I MASS Inc.), having a nominal frequency range from $10^{-4}$ $\mathrm{Hz}$ to $10^{4} \mathrm{~Hz}$. ${ }^{16}$ The sample was contained between a pair of $25 \mathrm{~mm}$ aluminum plates with a guard ring on the detector 
Table 1. Properties and Parameters (eq 12) of Linear and Three-Arm Star Polyisoprenes

\begin{tabular}{|c|c|c|c|c|c|}
\hline & $M_{w}$ & $M_{w} / M_{n}$ & $A(s)$ & B & $T_{\infty}$ \\
\hline 145 & 500 & 1.0 & $10^{-5}$ & 1302 & 17 \\
\hline L357 linear & 357000 & 1.08 & $6.72 \times 10^{-4}$ & 1302 & 170 \\
\hline S342 three-arm star & $342000^{a}$ & 1.10 & $5.58 \times 10^{-8}$ & 3429 & 95 \\
\hline
\end{tabular}

a Calculated from the molecular weight of the linear precursor, with the polydispersity determined from size exclusion chromatography.

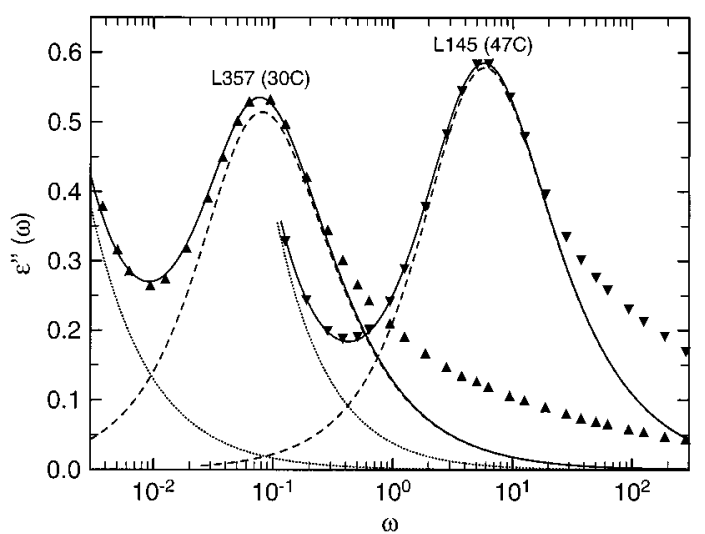

Figure 1. Representative terminal dispersions in the dielectric loss for the low $(\boldsymbol{\nabla})$ and high $(\boldsymbol{\Delta})$ molecular weight linear $\mathrm{PI}$, along with the best fit to eqs 1-3 (-). The dotted line represents the conductivity contribution (eq 2) and the dashed line the fitted KWW function (eq 3).

side. The gap size was typically $0.25 \mathrm{~mm}$. The temperature stability and uniformity within the sample chamber (Delta Design Model 9023) was better than $\pm 0.1 \mathrm{~K}$. Typically measurements were obtained at least twice at every temperature.

\section{Results}

A. Relaxation Function of Linear PI. Displayed in Figure 1 are representative normal mode dispersions in the dielectric loss for the two linear polyisoprenes. The relaxation contribution to the dielectric loss is given by the Laplace transform of the derivative of the decay function ${ }^{1,2}$

$$
\epsilon_{\text {rel }}(\omega)^{\prime \prime}=\epsilon_{\infty}+\Delta \epsilon \int_{0}^{\infty}-[\mathrm{d} \epsilon(\mathrm{t}) / \mathrm{dt}] \sin (\omega \mathrm{t}) \mathrm{dt}
$$

where $\Delta \epsilon$ and $\epsilon_{\infty}$ are respectively the dielectric strength and limiting high frequency permittivity, $\omega$ is circular frequency, and $t$ is time. Toward low frequency, the normal mode peak can be obscured by electrical conductivity, the contribution of which must be subtracted from the measured response. This conductivity can be described by ${ }^{17}$

$$
\epsilon_{\text {cond }}(\omega) \propto \omega^{-\mathrm{s}}
$$

where the exponent would equal unity for di rect current conductivity. Empirically, it has been found that $0.5 \leq$ $\mathrm{S} \leq 1$ for polyisoprene. ${ }^{13}$

For the normal mode decay, we use the Kohl rauschWilliam-Watts (KWW) stretched exponential equation $^{18}$

$$
\epsilon(\mathrm{t})=\exp -\left(\frac{\mathrm{t}}{\tau_{\mathrm{Kww}}}\right)^{\beta}
$$

in which $\tau_{\mathrm{K} w \mathrm{w}}$ is the relaxation time and $0<\beta \leq 1$. This function, originally derived as an empirical fitting function, is often used to analyze relaxation spectra.

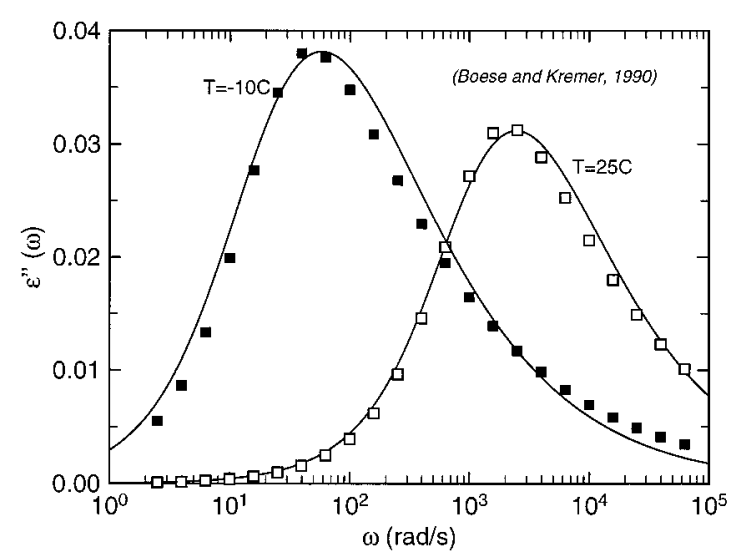

Figure 2. Normal mode data of Boese and $\mathrm{Kremer}^{13}$ for $\mathrm{PI}$ $\left(M_{w}=17000\right)$, al ong with the KWW function (eq 3) having the parameters obtained by fitting in the time domain the transform of their best-fit of the Havriliak-Negami function (eq 4) to the original dielectric loss data. Direct fitting of the dielectric loss to eqs 1 and 3 in the frequency regime would yiel $d$ a larger $\beta$ and more accurately represent the experimental points near the peak maximum.

Typical results of fitting eqs $1-3$ to the linear PI data are shown in Figure 1.

As is evident from Figure 1, the normal mode dispersion is quite narrow; the best-fit stretch exponent $(\beta)$ equals $0.87 \pm 0.01$. This is in accord with the limited data of Imanishi et al. ${ }^{7}$ on high molecular weight, monodisperse $\mathrm{PI}$, for which they reported a breadth at half-maximum (for the semilogarithmic pl ot) of slightly less than 1.5 decades. The KWW function does not fully describe the dielectric dispersions in Figure 1; in particular, there is a noticeable deviation at high frequencies. Before addressing the origin of this deviation, it is useful to note one consequence of it.

Boese and $\mathrm{Kremer}^{13}$ obtained dielectric spectra on $\mathrm{PI}$ over the frequency range from $10^{-1}$ to $10^{9} \mathrm{~Hz}$, which were fitted to the empirical Havriliak-Negami $(\mathrm{HN})$ equation ${ }^{19}$

$$
\epsilon(\omega)^{\prime \prime}=\frac{\Delta \epsilon \sin (\mathrm{b} \Psi)}{\left[1+2\left(\omega \tau_{\mathrm{HN}}\right)^{\mathrm{a}} \sin \left({ }^{1} / 2 \pi \mathrm{a}\right)+\left(\omega \tau_{\mathrm{HN}}\right)^{2 \mathrm{a}}\right]^{\mathrm{b} / 2}}
$$

where

$$
\tan \Psi=\frac{\left(\omega \tau_{\mathrm{HN}}\right)^{\mathrm{a}} \cos \left({ }^{1} / 2_{2} \pi \mathrm{a}\right)}{1+\left(\omega \tau_{\mathrm{HN}}\right)^{\mathrm{a}} \sin \left({ }^{1} / 2 \pi \mathrm{a}\right)}
$$

$\mathrm{a}$ and $\mathrm{b}$ are shape parameters, and the relaxation time $\tau_{\mathrm{HN}}>\tau_{\mathrm{KWw}}$. Boese and Kremer then transformed this analytical representation of the data to the time domain, whereupon direct fitting to eq 3 could be carried out. As has been shown previously, ${ }^{15,20}$ this method of analysis weights the tail of the dispersion, whereas fitting frequency domain data directly to eqs 1 and 3 emphasizes the peak. Hence, from the transform of the Havriliak-Negami fit to the dielectric loss data for a PI of $M_{w}=17000$ (the experiments of Boese and Kremer were limited to relatively low molecular weights due to conductivity interferences at the high frequencies used), a value of $\beta=0.56$ was obtained. ${ }^{13}$ As shown in Figure 2, this is less than the stretch exponent one would obtain by direct fitting of the Boese and Kremer data in the frequency domain.

The point is that the KWW fitting procedure is ambiguous when, as in the present situation, this 


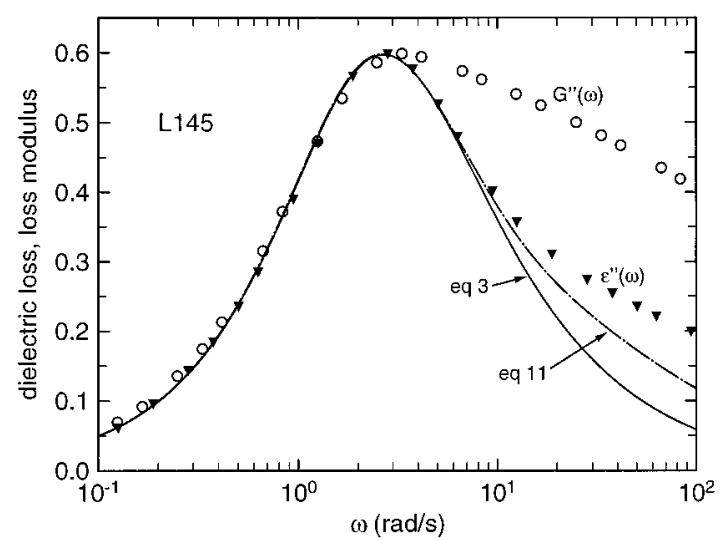

Figure 3. The terminal dispersion in the dielectric loss ( $\nabla$, measured at $\left.34{ }^{\circ} \mathrm{C}\right)$ and in the loss modulus $(\mathrm{O}$, taken from ref 15 ) for $L 145$, along with the best fit of respectively the KWW function $(-)$ and the coupling model including higher $p$ modes $\left(--_{-}\right)$to $\epsilon(\omega)^{\prime \prime}$. The mechanical data has been normalized to give the same peak intensity as the dielectric dispersion.

function does not completely describe the spectrum. Since our main interest is assessing the temperature dependence of the normal mode relaxation, the focus herein is on the center of the dispersion. This part of the terminal relaxation is less likely to be obscured by other contributions (e.g., from Rouse modes) than the higher frequency spectral wing.

Figures 3 and 4 depict the normal modes for the two linear PI. Also shown in these figures are the terminal dispersions in the mechanical loss modulus ${ }^{15}$ obtained on the same samples used herein for the dielectric measurements. Although the respective correlation functions are not identical, 10,11 mechanical and dielectric terminal spectra involve the same low-frequency chain motions. For both L 145 and L 357, the mechanical peak is much broader, particularly toward higher frequency. This agrees with the data of Adachi et al. ${ }^{21}$ for lower molecular weight PI.

The Rouse model for chain dynamics provides an inkling as to the origin of this difference. Valid for polymers of molecular weight too low for entanglement effects (i.e., lower than herein), the modulus of a Rouse chain is 22,23

$$
\mathrm{G}_{\text {Rouse }}(\mathrm{t}) \propto \sum_{\mathrm{p}=1}^{\infty} \exp \left(-\frac{\mathrm{p}^{2} \mathrm{t}}{\tau_{1}}\right)
$$

where $\tau_{1}$ is the relaxation time for the $p=1$ (slowest) mode. Note that all modes contribute equally to the mechanical response, al beit at different frequencies. The Rouse model yields for the dielectric normal mode relaxation 3,24

$$
\epsilon_{\text {Rouse }}(\mathrm{t}) \propto \sum_{\mathrm{p}, \text { odd }}^{\infty} \mathrm{p}^{-2} \exp \left(-\frac{\mathrm{p}^{2} \mathrm{t}}{\tau_{1}}\right)
$$

Due to the symmetry of type-A polymers, having an unreversed sequence of dipoles along the chain, even terms are absent from the series in eq 7. This, al ong with the $p^{-2}$ weighting of the odd terms, means that the longest $(p=1)$ mode dominates the dielectric spectrum. Of course, neither eq 6 nor 7 are applicable to the data in Figures 3 and 4, since the molecular weights of these two polymers are much greater than the entanglement molecular weight of $\mathrm{PI}\left(\mathrm{M}_{\mathrm{e}}=6400^{25,26}\right)$.

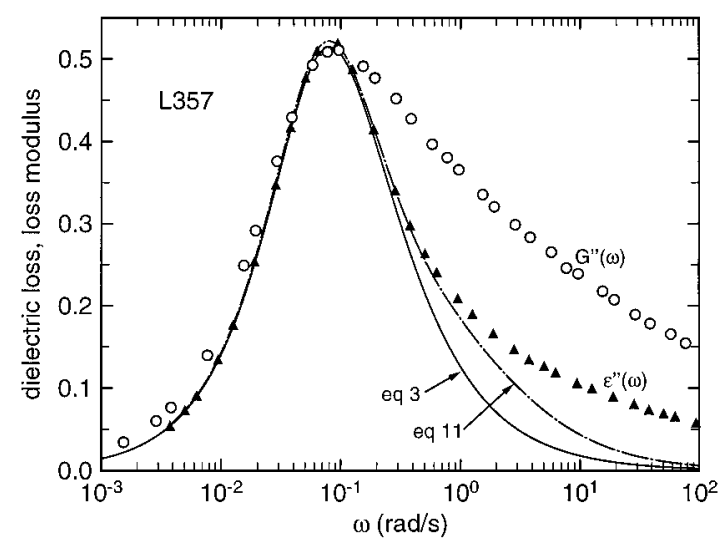

Figure 4. The terminal dispersion in the dielectric loss ( $\boldsymbol{\nabla}$, measured at $\left.30^{\circ} \mathrm{C}\right)$ and in the loss modulus $(0$, taken from ref 15$)$ for L357, along with the best fit of respectively the KWW function $(-)$ and the coupl ing model including higher $p$ modes $(---)$ to the dielectric data. The mechanical data has been normalized to give the same peak intensity as $\epsilon(\omega)^{\prime \prime}$.

Nevertheless, it is tempting to infer that the broader mechanical dispersion reflects the higher $(p>1)$ modes, the contribution of which is suppressed in the dielectric spectrum.

A more exact analysis requires that the entanglement effect be incorporated into the Rouse model. The bestestablished approach is to adopt the tube model, 22 based on the idea of chain reptation through topological constraints imposed by neighboring chains. ${ }^{27}$ The reptation prediction for the modulus is 22,23

$$
\mathrm{G}_{\text {reptation }}(\mathrm{t}) \propto \sum_{\mathrm{p}, \text { odd }}^{\infty} \mathrm{p}^{-2} \exp \left(-\frac{\mathrm{p}^{2} \mathrm{t}}{\tau_{\mathrm{d}}}\right)
$$

where the tube disengagement time, $\tau_{\mathrm{d}}$, is much longer than the longest Rouse time, $\tau_{1}$. In comparison to the Rouse model (eq 6), reptation predicts a narrowed distribution of relaxation times, a consequence of the one-dimensional motion of chains confined to an entanglement tube.22 It is well-known that experimentally the relaxation modulus is much broader than the reptation prediction. ${ }^{15,22}$

The loss of even modes and $\mathrm{p}^{-2}$ weighting, which in the dielectric spectra are a consequence of the symmetry of unreversed type-A dipoles, also is found in the tube model's expression for the modulus, leading to the expectation of equivalence of the dielectric and mechanical spectra for high molecular weight polymers. ${ }^{21}$ The contrary results in Figures 3 and 4 reflect the fact that strict confinement to a tube, whence the $\mathrm{p}^{-2}$ weighting and absence of even terms in eq 8 , is too restrictive an assumption.

Refinements of reptation theory have been proposed which include additional mechanisms, such as contour length fluctuations and constraint release. ${ }^{28-32}$ These modifications of the tube model serve to alleviate the severity of the tube constraints, allowing some chain diffusion transverse to the contour length. While the refinements improve the agreement with experiment, differences between the calculated and measured relaxation functions remain. Moreover, diel ectric data on PI dispersed in high molecular weight polybutadiene, wherein constraint release should be less important, are still at odds with the tube model. $8,10,12,33,34$

An alternative modification of the Rouse model accounts for entanglements by multiplying the Rouse relaxation rate by the quantity $\left(t / t_{c}\right)^{\beta-1}$, in which $t_{c}$ is 
the time scale over which the dynamics are unimpeded by intermolecular constraints. This approach is known as the coupling model (CM), ${ }^{35,36}$ since it emphasizes the intermolecular cooperativity of the dynamics in densely packed systems. Solving the rate equation for the relaxation of stress for coupled Rouse chains leads to 35,36

$$
\mathrm{G}_{\mathrm{CM}}(\mathrm{t}) \propto \sum_{1}^{\infty} \exp -\left(\frac{\mathrm{t}}{\tau_{\mathrm{p}}^{*}}\right)^{\beta}
$$

in which the coupled relaxation time of the pth mode is related to that of the corresponding Rouse mode as

$$
\tau_{\mathrm{p}}^{*}=\left[\tau_{1} \mathrm{p}^{-2} \mathrm{t}_{\mathrm{c}}^{\beta-1}\right]^{1 / \beta}
$$

While various approaches yield the first term in eq 9 for the relaxation function, ${ }^{37-41}$ eq 10 is unique to, and in fact the essence of, the coupling model. From eq 10, important predictions arise concerning the molecular weight and temperature dependence of the terminal relaxation of polymers. ${ }^{35,36,42,43}$ Since the terminal viscosity is determined primarily by the $p=1$ term, application of the coupling model heretofore employed the approximation of truncating the series in eq 9 to the first mode. Eq 9 then has the form of eq 3, which was used as an empirical fitting function by Tobolsky and co-workers ${ }^{44}$ and more recently by Palade et al. ${ }^{45}$ These investigators reported that it gave a reasonably good fit to the terminal relaxation modulus of entangled polymers.

In applying the coupling model to our dielectric data, we can retain the $p>1$ terms to yield from eqs 7 and 9

$$
\epsilon_{\mathrm{CM}}(\mathrm{t}) \propto \sum_{\mathrm{p}, \text { odd }}^{\infty} \mathrm{p}^{-2} \exp -\left(\frac{\mathrm{t}}{\tau_{\mathrm{p}}^{*}}\right)^{\beta}
$$

This assumes that the stretch exponent is independent of $p$, which should be okay for small $p$, representing modes with length scales much greater than the molecular weight between entanglements. Contributions beyond $\mathrm{p}=5$, which may be associated with an increasing $\beta$ (reflecting weaker topological constraints ${ }^{36,43}$ ) are negligible due to the $\mathrm{p}^{-2}$ weighting.

The fit of this expression to the measured dielectric loss data is shown in Figures 3 and 4 for L-145 and L357, respectively.

B. Temperature Dependence of Linear PI. Normal mode relaxation times were determined as the inverse of the frequency of the peak in the dielectric loss after subtraction of the conductivity. This yields values somewhat longer than the $\tau_{\mathrm{KWw}}$ in eq 3 . The results are shown in Figure 5, along with the corresponding terminal relaxation times determined mechanically on the same samples. ${ }^{15}$ All data conformed to the VogelFulcher equation 23

$$
\tau=\mathrm{A} \exp \frac{\mathrm{B}}{\mathrm{T}-\mathrm{T}_{\infty}}
$$

The parameters, tabulated in Table 1, enabled fits to the data over the limited temperature range of the measurements; as such, they cannot be relied upon for extrapolation. Additionally, given the phenomenological origin of the Vogel equation, ${ }^{23}$ no significance should be drawn from the respective values.

As shown in the figures, for both spectroscopies the data for the lower molecular weight PI, L 145, can be

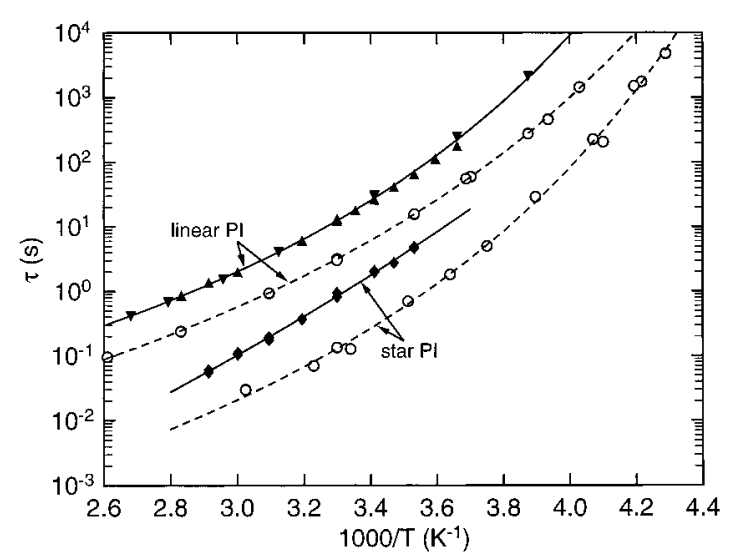

Figure 5. The dielectric normal mode relaxation times for the linear ( $\boldsymbol{\nabla}, \mathrm{L} 145 ; \mathbf{\Delta}, \mathrm{L} 357)$ and three-arm star ( $\downarrow$ ) PI, along with the corresponding mechanical relaxation times (hollow symbols) obtained on the same polymers (data taken from ref 15). The values for L 145 have been multiplied by a factor of 24 , which brings them into coincidence with the data for the higher molecular weight PI. The lines through the points represent the best-fit to eq 12 .

superimposed on the results for $L 357$ by multiplying the $\tau$ by a factor of 24; that is, the molecular weight dependence is the same for the two spectroscopies. This factor of 24 corresponds to a power dependence on molecular weight equal to $3.5 \pm 0.1$, which is consistent with earlier dielectric results ${ }^{6,13}$ and the molecular weight dependence of PI's viscosity. 49,50

The relaxation times determined dielectrically are 3.6 times longer than the mechanical values. This difference is consistent with the fact that the dielectric relaxation time corresponds to a mechanical retardation time $^{1}$ For a Maxwell model, the ratio of the retardation and relaxation times would equal the ratio of the unrelaxed and relaxed modulus (or dielectric constant); ${ }^{1}$ for polymers the relationship is more complicated.7,11 As an aside, we note that the dielectric and mechanical segmental relaxation times were found to differ by a factor of 20 for polyisoprene. ${ }^{51}$

It can also be seen in Figure 5 that the temperature dependence of the dielectric and mechanical relaxations are the same, notwithstanding that the latter has a broader relaxation function (e.g., Figures 3 and 4). There is a well-established empirical correlation between the shape of a relaxation function and the temperature dependence of the relaxation time, with broader dispersions exhibiting greater temperature sensitivity. ${ }^{43,52-58}$ The absence of such a correlation in the present situation is due to the fact that the same relaxation is being measured by the two spectroscopies. The additional breadth seen in the mechanical spectrum reflects the contribution of additional modes (cf. eqs 6 and 7), rather than any inherently different relaxation behavior.

C. Three-Arm Star PI. Previous mechanical measurements ${ }^{15}$ on the three-arm star PI, S342, revealed its terminal dispersion to be broader and, more significantly, the temperature sensitivity of its terminal relaxation time greater than for linear PI. Since the normal mode relaxation for a polymer with unreversed dipole moments parallel to the chain can be identified with the end-to-end displacement, we anticipate at least qualitatively the same behavior for the dielectric spectroscopy results.

Figure 6 shows the dielectric loss measured for the S342. After subtraction of the conductivity (eq 2), the normal mode peak is obtained. The range of tempera- 


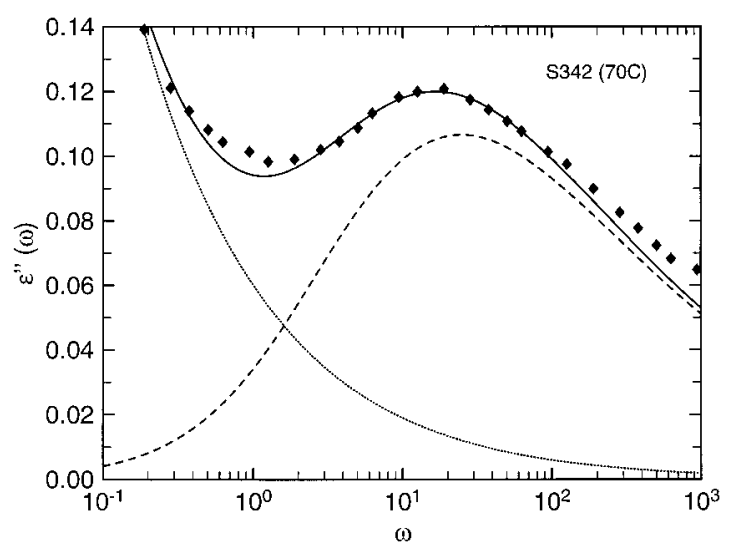

Figure 6. Normal mode for three-arm star PI ( $\bullet$ ), along with the best fit to eqs $1-3(-)$. The dotted line represents the conductivity contribution (eq 2) and the dashed line the KWW function. Partly due to polydispersity, the fit to eq 3 is mediocre.

tures over which acceptable measurements could be obtained on the star PI was limited by interferences from conductivity. Although the inherent conductivity is not worse for the star in comparison to the linear PI, the former's broader dispersion makes it more difficult to accurately extract the relaxation component from the experimental measurements. Nevertheless, as seen in Figure 6, the dielectric loss peak for S342 is > 1 decade broader than the normal mode peak measured for the linear PI (Figures 3 and 4). Although conforming to eq 3 only toward the lower frequencies, we estimate for the three-arm star PI that $\beta=0.42 \pm 0.04$, compared to the result for linear $\mathrm{PI}, \beta=0.87 \pm 0.01$.

The normal moderelaxation times for S342 are shown in Figure 5, al ong with the data for the linear PI. Apart from any contribution from other mechanisms, the temperature dependencies of linear and branched polymers are predicted by reptation theory to be equivalent, because the model has only one friction factor.22,59 Since the plots in Figure 5 are not linear, the respective temperature sensitivities cannot be assessed directly from the slopes of the Arrhenius curves.

A method to compare temperature dependencies of linear and branched polymers has been suggested by Graessley, ${ }^{60,61}$ based on the idea that a branched chain requires retraction of its arm in order to proceed along its "tube" of constraints. 27,62 Such retraction produces a more compact transient structure. This corresponds to a higher concentration of gauche conformers, the higher energy of which would produce a higher activation barrier for the terminal relaxation process. Polyisoprene is exceptional, however, in that its trans and gauche conformers have the same energy. ${ }^{61}$ Accordingly, PI is predicted to be unique, having the same temperature dependence, independent of branch structure. ${ }^{50,60,61}$

To test this idea, in Figure 7 we display the normal mode relaxation times for the stars normalized by the relaxation times for the linear PI (using eq 12 for interpolation). Although there is some scatter in the results, reflecting uncertainty in the conductivity subtraction, it is clear that the three-arm star exhibits a stronger temperature dependence than the linear PI. From the slope in Figure 7, we estimate that the extra temperature dependence of the star corresponds to an activation energy of $1.3 \pm 0.2 \mathrm{kcal} / \mathrm{mol}$. Thus, the experimental results are in qualitative disagreement with the prediction of reptation theory for the relative temperature dependencies of linear and star PI.60,61

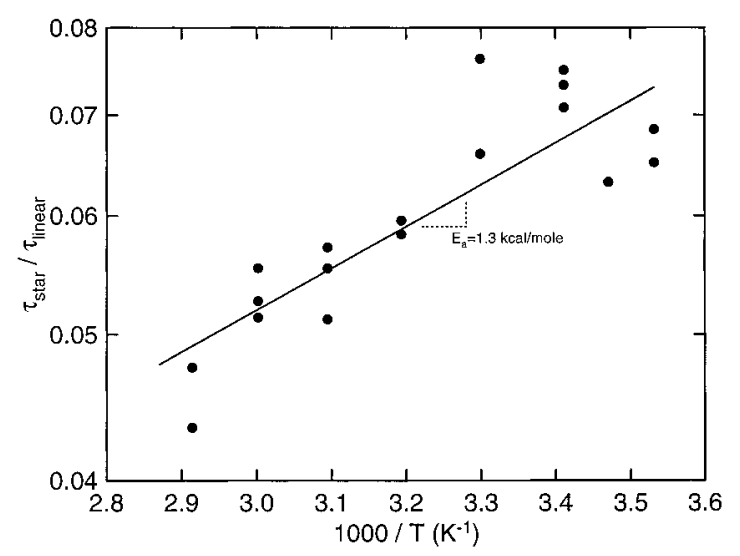

Figure 7. Arrhenius plot of the ratio of the normal mode relaxation times for the three-arm star S342 and the linear PI (see Figure 5). The slope yields a value for the excess activation energy, equal to $1.3 \mathrm{kcal} / \mathrm{mol}$.

These dielectric results corroborate previous mechanical data showing that $\mathrm{PI}$ is quite similar to other polymers with respect to the effect of branching on its temperature dependence. I nterestingly, the mechanical result for S342 indicated an excess activation energy equal to $2.3 \mathrm{kcal} / \mathrm{mol},{ }^{15}$ Iarger than the dielectric value. However, branched PI exhibits thermorheological complexity in the terminal region of its viscoelastic spectrum, ${ }^{15}$ which is the usual situation for branched polymers. ${ }^{60,61}$ Since the terminal zone changes shape with temperature, a different measure of temperature dependence (rather than comparing peak frequencies) would yield a different excess activation energy in Figure 7. Finally, we point out that the stronger temperature dependence of the branched $\mathrm{PI}$ is not related to the magnitude of its local friction coefficient, as evidenced by the equal glass transition temperatures of the linear and star PI.15

\section{Summary}

Dielectric spectroscopy offers advantages for the study of the low-frequency dynamics of polymeric liquids. Dielectric spectra reflect the same chain motions as the mechanical modulus; however, the reduced interference, due to symmetry, from shorter time processes (i.e., the higher Rouse modes in eqs 6 and 7) serves to isolate the normal mode. Better resolution of the terminal dispersion facilitates assessment of rheological models.

This advantage of dielectric spectroscopy for analysis of the terminal mode is shared by mechanical compliance measurements. ${ }^{63}$ Unfortunately, theoretical models invariably provide expressions for the relaxation modulus, which usually cannot be obtained analytically from compliance data. For example, there is no analytical representation of the compliance function for the Rouse model. ${ }^{64}$ This accounts for the preference for the modulus rather than the compliance. However, the dielectric permittivity provides a direct measure of the decay function (eq 1), as well as emphasizing the terminal mode.

Herein the dielectric normal mode of PI was seen to be significantly narrower than the corresponding dispersion in the loss modulus. While this is expected for unentangled polymers, it was found to be the case even for PI having a molecular weight more than 50 times greater than $\mathrm{M}_{\mathrm{e}}$. Such an observation is at odds with the tube model's description of the long-range dynamics in terms of reptation al ong a tube of fixed constraints. The fact that the tube model is overly restrictive in this 
regard has been established from mechanical relaxation measurements. ${ }^{28-32}$

The present dielectric results confirm previous mechanical data showing the terminal relaxation times of star-branched PI to be more sensitive to temperature than linear PI. This is at odds with reptation theory, in which the only temperature-dependent quantities are the local friction factor and, when arm-retraction mechanisms become important, a transition state energy. 22,59-61 Since for PI there is no energy difference between gauche and trans rotamers, the reptation prediction is that no difference in temperature dependence between linear and star PI exists 60,61

The coupling model of relaxation adopts a strategy diametrically opposite to that of a single chain theory like reptation, in that molecular considerations are ignored, with the emphasis placed on the general principles governing constraint dynamics in condensed matter. The coupl ing model makes the opposite prediction from reptation concerning the temperature dependence of branched polymers. An explicit prediction of the coupling model is that broader relaxations will always be more sensitive to temperature. In this respect, the coupling model correctly describes the relative temperature dependencies of linear and star PI.

Acknowledgment. We thank K. L. Ngai and P. G. Santangelo for insightful discussions. This work was supported by the Office of Naval Research. C.A.B. expresses his appreciation to the National Research Council for a postdoctoral fellowship.

\section{References and Notes}

(1) McCrum, N. G.; Read, B. E.; Williams, G. Anelastic and Dielectric Effects in Polymeric Solids, Dover Publications: New York, 1967.

(2) Riande, E.; Saiz, E. Dipole Moments and Birefringence of Polymers, Prentice Hall: Englewood Cliffs, NJ , 1992.

(3) Stockmayer, W. H. Pure Appl. Chem.1967, 15, 539.

(4) Schallamach, A.; Thirion, P. Trans. Faraday Soc. 1949, 45, 605.

(5) Norman, R. Proc. Inst. Elec. Eng. (London) 1953, 100, 41.

(6) Adachi, K.; Kotaka, T. Macromolecules 1984, 17, 120; 1985, $18,466$.

(7) Imanishi, Y.; Adachi, K.; Kotaka, T. J . Chem.Phys. 1988, 89, 7585; 7593.

(8) Adachi, K.; Nakamoto, T.; Kotaka, T. Macromolecules 1989, $22,3111$.

(9) Adachi, K.; Wada, T. Kawamoto, T. Kotaka, T. Macromolecules 1995, 28, 3588.

(10) Watanabe, H.; Yamazaki, M.; Yoshida, H.; Adachi, K.; Kotaka, T. Macromolecules 1991, 24, 5365; 5372.

(11) Watanabe, H.; Yao, M.-L.; Osaki, K. Macromolecules 1996, 29, 97.

(12) Watanabe, H.; Urakawa, O.; Yamada, H.; Yao, M.-L. Macromolecules 1996, 29, 755 .

(13) Boese, D.; Kremer, F. Macromolecules 1990, 23, 829.

(14) Boese, D.; Kremer, F.; Fetters, L. J . Macromol ecules 1990, 23, 1826.

(15) Bero, C. A.; Roland, C. M. Macromolecules 1996, 29, 1562.

(16) Mopsik, F. I. Rev. Sci. Instrum. 1984, 55, 79.

(17) Mott, N. F.; Davis, E. A. Electronic Processes in Non Crystalline Materials; Clarendon Press: Oxford, 1979.

(18) Williams, G.; Watts, D. C. Trans. Faraday Soc. 1970, 66, 80.
(19) Havriliak, S.; Negami, S. Polymer 1967, 8, 161

(20) Roland, C. M.; Santangelo, P. G.; Ngai, K. L.; Meier, G. Macromol ecules 1993, 26, 6164.

(21) Adachi, K.; Yoshida, H.; Fukui, F.; Kotaka, T. Macromolecules 1990, 23, 3138.

(22) Doi, M.; Edwards, S. F. The Theory of Polymer Dynamics, Clarendon Press: Oxford, 1986.

(23) Ferry, J . D. Viscoel astic Properties of Polymers, Wiley: New York, 1980.

(24) Zimm, B. H. J . Chem. Phys. 1956, 24, 269.

(25) Gotro, J. T.; Graessley, W. W., Macromolecules 1984, 17, 2767.

(26) Roland, C. M. Macromolecules 1992, 25, 7031

(27) de Gennes, P. G. J . Phys. (Paris) 1975, 36, 1199.

(28) Graessley, W. W. Adv. Polym. Sci. 1982, 47, 67.

(29) Doi, M. J. Polym. Sci. Polym. Phys. Ed.1983, 21, 667.

(30) Rubenstein, M.; Colby, R. J . Chem. Phys. 1988, 89, 5291.

(31) des Cloizeaux, J. Macromolecules 1990, 23, 4678.

(32) Viovy, J . L.; Rubenstein, M.; Colby, R. Macromol ecules 1991 24, 3587.

(33) Watanabe, H.; Urakawa, O.; Kotaka, T. Macromol ecules 1993, 26, 5073.

(34) Watanabe, H.; Yamada, H.; Urakawa, O. Macromolecules 1995, 28, 6443.

(35) McKenna, G. B.; Ngai, K. L.; Plazek, D. J . Polymer 1985, 26, 1651.

(36) Ngai, K. L.; Rendell, R. W.; Rajagopal, A. K.; Teitler, S. Ann. N.Y. Acad. Sci. 1986, 484, 150

(37) Cohen, M. H.; Grest, G. S. Phys. Rev. B 1981, 24, 4091.

(38) Cohen, M. H.; Grest, G. S. Phys. Rev. B 1982 26, 2664.

(39) Schlesinger, M. F.; Montroll, E. W. Proc. Natl. Acad. Sci. U.S.A. 1984, 81, 1280.

(40) Palmer, R. B.; Stein, D. L.; Abrahams, E.; Anderson, P. W. Phys. Rev. Lett. 1984, 53, 958.

(41) Ogielski, A. T.; Stein, K. L. Phys. Rev. Lett. 1985, 55, 1634.

(42) Ngai, K. L.; PLazek, D. J . J . Polym. Sci. Polym. Phys. Ed. 1986, 24, 619.

(43) Ngai, K. L.; Plazek, D. J . Rubber Chem. Tech.-Rubber Rev. 1995, 68, 376.

(44) Knoff, W. F.; Hopkins, I. L.; Tobolsky, A. V. Macromolecules 1971, 4, 750 .

(45) Palade, L. I.; Verney, V.; Attane, P. Macromolecules 1995 28, 7051.

(46) Roland, C. M.; Ngai, K. L. J . Chem. Phys. 1996, 104, 2967.

(47) Roland, C. M.; Ngai, K. L.; Lewis, L. J . J . Chem. Phys. 1996, $104,4632$.

(48) Roland, C. M.; Ngai, K. L. J . Chem. Phys. 1995, 103, 1152

(49) Pearson, D. S.; Mueller, S. J .; Fetters, L.J .; Hadjichristidis, N. J . Polym. Sci., Polym. Phys. Ed. 1983, 21, 2287.

(50) Fetters, L. J .; Kiss, A. D.; Pearson, D. S.; Quack, G. F.; Vitus, F. J . Macromolecules 1993, 26, 647.

(51) Colmenero, J .; Alegria, A.; Santangelo, P. G.; Ngai, K. L.; Roland, C. M. Macromolecules 1994, 27, 407.

(52) Roland, C. M.; Ngai, K. L. Macromolecules 1991, 24, 5315; 1992, 25, 1844.

(53) Ngai, K. L.; Roland, C. M. Macromolecules 1993, 26, 6824.

(54) Roland, C. M.; Ngai, K. L. Macromolecules, 1992, 25, 5765.

(55) Torell, L. M.; Grimsditch, M. Proc. Phys. 1989, 37, 196.

(56) Plazek, D. J .; Ngai, K. L. Macromolecules 1991, 24, 1222.

(57) Bohmer, R.; N gai, K. L.; Angell, C. A.; Plazek, D. J . J . Chem. Phys. 1993, 99, 4201.

(58) Roland, C. M. Macromolecules 1994, 27, 4242.

(59) Graessley, W. W. Acc. Chem. Res. 1977, 10, 332.

(60) Carella, J. M.; Gotro, J . T.; Graessley, W. W. Macromolecules 1986, 19, 659.

(61) Graessley, W. W. Macromolecules 1982, 15, 1164.

(62) Doi, M.; Kuzuu, N. Y. J . Polym. Sci. Polym. Lett. Ed. 1980, $18,775$.

(63) Plazek, D. J . J . Rheol. 1992, 36, 1671.

(64) Berry, G. J. Polym. Sci. Polym. Phys. Ed. 1987, 25, 2203. MA960099N 